\title{
Proposta de um Ambiente Colaborativo para a Coordenação de Cuidado de Pacientes Crônicos
}

\author{
Márcia Ito ${ }^{1,2}$, Débora Lina Nascimento Ciriaco Pereira ${ }^{1,2}$ \\ ${ }^{1}$ IBM Research Brasil - São Paulo - SP - Brazil \\ ${ }^{2}$ Departamento de Informática em Saúde - Escola Paulista de Medicina - Universidade \\ Federal de São Paulo - São Paulo - SP - Brazil \\ $\{$ marciaito, dlina\} $@ b r . i b m . c o m$
}

\begin{abstract}
The CPRM model (Chronic Patient's Relationship Management) uses the CRM concepts (Customer Relationship Management) to manage the relationship between the patient and his doctor to improve health care. However, care coordinate activities (care coordination) of the professionals that assist the patient (care team) is important to increase patient access to health services. Thus, we decided to extend the CPRM model to carry the relationship management of the care team: a collaborative environment that helps coordinate the patients care activities, using and sharing information throughout the patient care team, the patient and his family.
\end{abstract}

Resumo. O modelo GRPC (Gestão do Relacionamento do Paciente Crônico) usa os conceitos de CRM (Customer Relationship Management) na gestão do relacionamento entre o paciente e seu médico para melhorar a assistência médica. Porém, coordenar as atividades de assistência (coordenação de cuidado) realizadas pelos profissionais que atendem o paciente (time de cuidado) é tão importante quanto aumentar o acesso do paciente aos serviços de saúde. Assim, estendeu-se o modelo GRPC para comportar a gestão do relacionamento do time de cuidado: um ambiente colaborativo que ajude a coordenar a assistência dos pacientes, usando e compartilhando a informação por todo o time de cuidado, o paciente e seus familiares.

\section{Introdução}

As doenças crônicas são um dos grandes desafios da saúde e do desenvolvimento econômico no século XXI, pois estão relacionadas diretamente com a qualidade de vida da população, assim como com a produtividade e os gastos mundiais. Tem-se que, anualmente, aproximadamente 14 milhões de pessoas entre 30 a 70 anos morrem devido a doenças crônicas. Estima-se ainda uma perda de US\$ 7 trilhões em produtividade e gastos em saúde, nos próximos 20 anos, caso nenhuma medida seja tomada. Por isso, a Organização Mundial de Saúde (OMS) vem elaborando estratégias para diminuir este números. Dentre as estratégias tem-se que a tecnologia da informação e comunicação exerce importante papel, mostrando-se parte da solução, ao facilitar o acesso e a troca de informação entre profissionais, pacientes e cuidadores, além de auxiliar na comunicação/interação entre eles. [WHO 2014] [Ito 2008] Por conta desta 
necessidade Ito [Ito 2008] desenvolve o modelo de Gestão do Relacionamento do Paciente Crônico (GRPC) que permite fornecer um atendimento centrado no paciente ao incentivar a gestão do relacionamento deste com o seu médico, por meio de acompanhamento personalizado. Aliás, pacientes crônicos geralmente possuem um diagnóstico principal e outros secundários, exigindo atendimento por mais de uma especialidade médica, além de outros profissionais que orientam em relação a mudanças de comportamentos necessários à sua saúde como nutricionistas e fisioterapêutas. Os profissionais que atendem a um mesmo paciente formam uma equipe denominada time de cuidado. Coordenar as atividades de assistência destes profissionais é essencial para o otimizar o uso dos recursos de saúde, neste trabalho, será adotado o termo coordenação de cuidado para designar tal coordenação. Ademais, de acordo com Owens [Owens 2010] os gastos aproximados de um paciente em que não existe uma coordenação de cuidado é $75 \%$ mais alto do que daqueles que as tem. $\mathrm{O}$ autor sugere ainda que, ao melhorar a coordenação de cuidado pode-se diminuir em $35 \%$ os custos com o paciente. Isto posto, decidiu-se estender o modelo GRPC para realizar também a gestão do relacionamento entre os participantes do time de cuidado.

\section{Modelo GRPC e a Coordenação de Cuidado: Modelo GRPC estendido}

O conceito de gerenciamento da saúde do paciente por meio de equipes coordenadas ao cuidado tem sido explorado por diversos autores desde o fim da década de 1960. Várias definições têm sido propostas, sendo a mais citada a do estudo sobre Coordenação de Cuidado (Care Coordination), realizado pela universidade de Stantford, em 2007, na qual a coordenação de cuidados ${ }^{1}$ é definida como: "[...] organização deliberada das atividades de cuidado do paciente, realizadas por dois ou mais participantes envolvidos no processo de cuidado (incluindo o paciente), a fim de facilitar a entrega apropriada dos serviços de saúde. Sendo a organização de cuidado tanto a mobilização de pessoal quanto de outros recursos necessários para realizar todas as atividade de atendimento ao paciente. Essa, muitas vezes gerida pela troca de informações entre os participantes responsáveis pelos diferentes aspéctos do cuidado." [McDonald 2007] A coordenação de cuidado tem como objetivo diminuir a fragmentação do cuidado e melhora a entrega dos serviços de saúde. Deste modo, existem vários esforços para implementar este tipo de programa [McDonald 2007]. Estudos realizados pelo Medicare [Craig 2011] em 2007 demonstraram que a maioria dos programas de coordenação de cuidado praticado pelas instituições de saúde americanas falharam. Concluiram que os que tiveram sucesso tinham uma característica comum, o relacionamento entre o coordenador de cuidado (care coordinator) e o paciente ia além do serviço médico. O coordenador conhecia as necessidades dos pacientes e conectava-se a ele de forma pessoal. Assim, o estudo conclui que o relacionamento entre o coordernador e o paciente é importante, pois o tratamento envolve mudança de comportamento e escolhas que estão sob o controle do paciente. Neste ponto, o coordenador tem fundamental importância em suas decisões, pois ele age como conselheiro. Além desse, outros estudos [Wagner 2014] [Houdt 2014] indicaram que os programas de sucesso são aqueles em que se constroem relacionamentos duradouros e de confiança entre o paciente e o time de cuidado e entre os membros do time de cuidado. Por outro lado, o modelo GRPC original foi

\footnotetext{
${ }^{1}$ Tradução feita pelos autores e transcrita
} 
desenvolvido para assistir ao paciente crônico permitindo o controle adequado da doença, baseado nas melhores práticas e no contexto psicossocial do paciente. Assim, ao adaptar a arquitetura da tecnologia CRM pode-se criar um aplicativo que integra o prontuário eletrônico do paciente, os sistemas de apoio à decisão e as soluções de telemedicina, para permitir o monitoramento personalizado. Todavia, o modelo contemplava apenas a gestão do relacionamento entre o médico e o paciente, assim se o paciente fosse acompanhado por mais de uma especialidade não havia uma coordenação de suas atividades assistênciais. Por exemplo, o sistema poderia verificar a existência de uma interação medicamentosa e alertar os médicos, porém não havia uma forma prevista pelo modelo da comunicação entre os dois profissionais para decidir o que fazer em relação a interferência do medicamento no tratamento global do paciente. Assim, viu-se a necessidade de estender o modelo para que contemplasse algum tipo de coordenação do cuidado. Estudos sobre programas de coordenação do cuidado demonstraram que ter uma gestão do relacionamento entre o time de cuidado e o paciente e entre os membros do time são premissas para o seu sucesso. Neste ponto, o Modelo GRPC, tendo como conceito a interatividade, confiança, conscientização, credibilidade e orientações personalizadas defendendo um relacionamento de parceria entre o médico e o paciente possui os critérios necessários para um adequado modelo de coordenação do cuidado, bastando acrescentar a gestão do relacionamento do time de cuidado do paciente como forma de suprir a demanda encontrada. Neste modelo o coordenador de cuidado é o administrador do sistema. Uma vez o paciente inscrito para o programa, o coordenador define o time de cuidado no sistema e a partir desse momento passa a mediar o acompanhamento do paciente pelo time. Para implementar o novo modelo foi necessário estender a arquitetura na qual os conceitos do CRM do modelo GRPC original foram mantidos e acrescentou-se a coordenação de cuidado do paciente. Surgiu assim um ambiente colaborativo para o acompanhamento do paciente e do time de cuidado. Neste ambiente colaborativo o paciente interage com os membros da sua equipe de cuidado e com o coordenador, assim como o coordenador com o time de cuidado e os membros do time comunicam-se entre si.

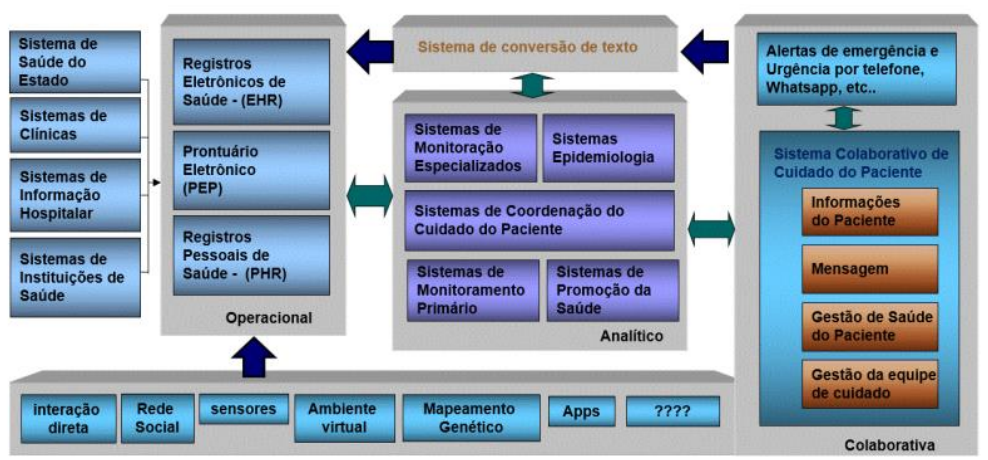

Figura 1. Arquitetura do Modelo GRPC estendido

A arquitetura estendia (figura 1) possui: (1) o módulo operacional que contém (a) o Registro Eletrônico de Saúde (EHR) que armazena os dados administrativos do paciente como por exemplo os custos do tratamento realizado; (b) o Prontuário Eletrônico do Paciente (PEP) que contém os dados do prontuário do paciente possuindo uma confiabilidade maior para a análises posteriores e (c) os Registros Pessoais de Saúde (PHR) que são as informações fornecidas pelo paciente e logo com confiabilidade 
menor, porém importante para alertar o time de cuidado; (2) o módulo análitico que acrescenta o sistema de coordenação de cuidado do paciente aos que já existiam no modelo GRPC e (3) O módulo colaborativo que agora tem um sistema para a gestão de saúde do paciente (relacionamento entre o paciente e o time, além do coordenador do cuidado) e a gestão da equipe de cuidado (relacionamento entre os membros do time e do coordenador com o time) que é denominado sistema colaborativo de cuidado do paciente. Este sistema baseia-se em modelos de redes sociais os quais facilitarão a análise dos dados extraídos da interação entre seus usuários. O restante do módulo colaborativo permanece com as que já existiam no modelo GRPC original.

\section{Discussão e Trabalhos Futuros}

Este artigo apresentou a extensão do modelo GRPC que acrescenta a coordenação de cuidado do paciente, prática que vem cada vez mais sendo utilizada como forma de melhorar a qualidade da assistência à saúde. Vários estudos demonstram que um modelo de sucesso compreende aquele em que o relacionamento entre as pessoas deve ser de parceria incluindo um coordenador, o paciente e os profissionais que o atendem. Ao estender o modelo GRPC, propôs-se um ambiente colaborativo baseado em modelos de redes sociais. O objetivo é que este ambiente facilite a coordenação de cuidado do paciente, melhore a visualização das informações do paciente pelo time de cuidado, melhore a compreensão do fluxo dos pacientes e demanda dinâmica sobre a estrutura de serviços, otimize o uso de recursos dos diferentes serviços e o auxilie no desenvolvimento de critérios e métricas de qualidade do serviço, do atendimento e da aderência do paciente ao seu tratamento. Um protótipo deste ambiente está em desenvolvimento e espera-se avaliar o uso deste ambiente na coordenação do cuidado dos pacientes de uma Instituição de Saúde Especializada em Oncologia Pediátrica na qual atualmente tal coordenação é feita de forma manual.

\section{Referências}

Craig, C., Eby, D., Whittington J. (2011) "Care Coordination Model: Better Care at Lower Cost for People with Multiple Health and Social Needs". IHI Innovation Series white paper. Cambridge, Massachusetts: Institute for Healthcare Improvement.

Houdt, V., et al (2014) "Focus groups to explore healthcare professionals' experiences of care coordination: towards a theorical framework for the study of care coordination" BMC Family Practice. 15:177

Ito, M., Martini, J. S. C., lochida, L. C. (2008) "CPRM: A Chonic Patient's Management Model Based on the Concepts of Customer's Relationship". In: Proceedings of the 2008 ACM Symposium on Applied Computing. ACM New York: NY. Pages: 1364-1368

McDonald K.M., et al. (2007) Care Coordination. Vol 7. Closing the Quality Gap: A Critical Analysis of Quality Improvement Strategies. Rockville, MD: Agency for Healthcare Research and Quality.

Owens, M. K. (2010). Costs of uncoordinated Care. In P. L. Yong, R. S. Saunders, \& L. A. Olsen (Eds.). The healthcare imperative: Lowering costs and improving outcomes: Workshop series summary. Washington, DC: National Academy Press. (109-140).

Wagner, E. H. et al. (2014) Improving care coordination in primary care. Med Care. 52(11 Suppl 4): S33-8.

WHO - World Health Organization (2014), Global Status Report on noncommunicable diseases 2014, WHO: Switzerland. 324 p. 